\title{
THE INFLUENCE OF OCCLUSION ON MASTICATORY EFFICIENCY CONSIDERING RELEVANT INFLUENCING FACTORS
}

\author{
Gregor Slavicek ${ }^{1,2, a^{*}} \odot$ \\ 1Steinbeis Transfer Institute Biomedical Interdisciplinary Dentistry, Steinbeis University Berlin, DE-12489 Berlin, Germany \\ 20REHAB-Minds GmbH, DE-70567 Stuttgart, Germany \\ aMD, DDS, MSc, Director and Head, CE0 and Head; e-mail: gregor.slavicek@stw.de; ORCIDiD: https://orcid.org/0000-0003-2454-4048
}

Introduction The role of the occlusal quality as a relevant factor for mastication is controversially discussed. This paper aims to determine the role of the occlusion given the influencing factors. The correlation between the subjective evaluation of treatment needs and objective chewing test results are assessed.

Methodology 842 participants (female $n=460,54.5 \%$; male $n=382,45.4 \%$ ) performed a standardized chewing test. The participants' occlusal quality, angle classification, age, gender, treatment needs and intraoral status were recorded. The participants were instructed to break down the standardized chewing tests units. The particles were collected in a sieve and placed on a calibrated acquisition board. Standardized images were analysed, measuring the areas of each particles in $\mathrm{mm}^{2}$. Null Hypotheses were tested with the Kruskal-Wallis tests and post hoc tests with Bonferroni correction, to be rejected at $p \leq 0.01$.

Results The occlusion quality has a significant impact on the chewing efficiency ( $p \leq 0.001$ ), but angle classification only to some extent. Age $(p \leq 0.001)$ and gender $(p \leq 0.001)$ are important intrinsic factors. Fixed prosthodontics do not reach the chewing performance of natural occlusion $(p \leq 0,01)$. The subjective clinical assessment of treatment needs correlates in categories with strong differentiations $(p \leq 0,001)$, but not if only minor differences are asserted ( $p \leq 0,515)$.

Conclusion The occlusion quality has a significant impact on the chewing efficiency. Masticatory performance is highly dependent on the natural or artificial chewing surface morphology. Age, gender and the intraoral status are important intrinsic factors. The maintenance of a sufficient functional oral status is a crucial task in the care of the aging population.

\section{KEYWORDS}

Chewing Efficiency; Chewing Surface Morphology; Dental Occlusion; Mastication; Standardized Chewing Test.

\section{INTRODUCTION}

\subsection{Aim of mastication}

The aim of a masticatory process is to comminute food as homogeneously as possible, to moisten the food bolus with saliva and to prepare it for swallowing. The principle of the mechanical processing of food is found in all mammals, including humans. From an evolutionary perspective, it is an advantage to comminute food prior to digestion in the gastrointestinal tract. This mechanical fragmentation makes digestion more efficient [1]. The provision of energy and essential nutrients can be guaranteed by the regular intake of smaller, but mechanically processed amounts of foods. The masticatory process poses a major functional challenge to the stomatognathic system for its whole lifetime [2]. Mastication exerts a substantial effect on human health [3]. Food, inserted in the mouth, is normally known. So, an expectation 


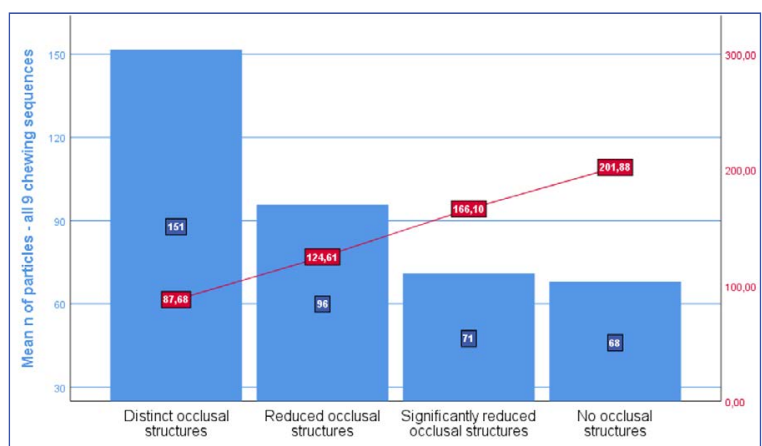

Figure 1a. Overall chewing efficiency vs. occlusal quality.

exists, reinforced by visual, olfactory, and gustatory sensations. Based on this preliminary information, patterns can be retrieved from memory that are optimally adapted to the food. If the feedback from the intra- and extraoral proprioceptors differs from the expectations, the masticatory pattern will be adapted [4]. These feed-back mechanisms react to the so-called mouth feel of food (Tab.1).

\subsection{Teeth are highly specialized tools}

Teeth are used as highly specialised tools when food needs to be comminuted. Teeth have adapted over the course of evolution to the needs of the mammalian species in an astonishing variety of forms [5]. A food bolus positioned between the dental arches is broken down mechanically. The structures of the occlusal surface of the teeth tend to be flat with prominent enamel ridges in many mammals, so the masticatory forces have an optimal effect on food [6]. The huge variety in the morphology of the masticatory surfaces can be explained by adaptation to extrinsic factors [7].

Just like tooth morphology, the shape of the occlusal surfaces, and the spatial arrangement of the teeth differ considerably in many species, oversimplified traceable on the carnivores-herbivores differences. The teeth of carnivores (meat-eaters) are completely different from the teeth of herbivores (plant-eaters) [8]. Catching and retaining prey are tasks that require considerable strength and robustness of the carnivores stomatognathic system. The teeth of carnivores chisel off large pieces, which are then swallowed entirely. The actual process of digestion is performed in the digestive tract. Herbivores have totally different demands on teeth and the chewing organ. Leaves and twigs can be grasped easily. Excessive mechanical processing is performed in the oral cavity. The tooth surfaces are moved against one another in a distinct direction - so plant fibres are mechanically comminuted. The direction of chewing movements is adapted to the structure of the tooth surfaces [4].

Unphysiological chewing movements may have serious effects on teeth, periodontium, and the neuromuscular system [9]. The relationship with craniomandibular dysfunctions (CMD) is also a subject of discussion [10]. A preferred chewing side

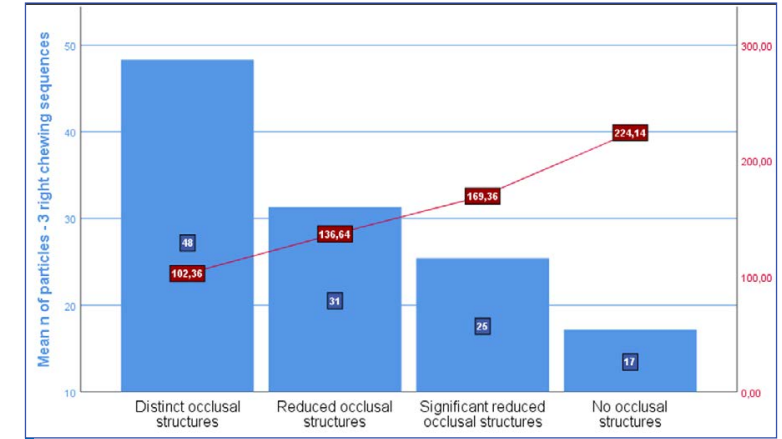

Figure 1b. Right side chewing efficiency vs. occlusal quality of the right side.

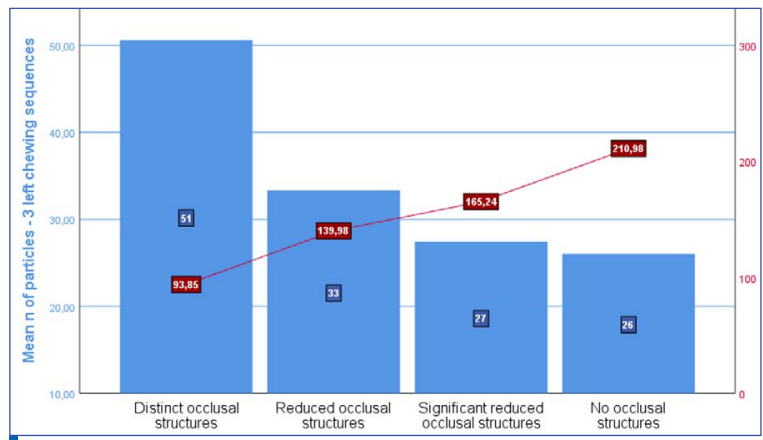

Figure 1c. Left side chewing efficiency vs. occlusal quality of the left side.

and a reduced chewing ability is reported more often in patients with CMD symptoms [11]. The masticatory patterns of children with deciduous teeth are characterised by wide lateral movements to the working side on opening and a more inwardly directed path during the closing phase of the masticatory cycle. In adults, on the other hand, the opening movement are performed in the median-sagittal plane. The closing movement is characterised by a wide lateral movement to the working side. The closing paths of the molars on the working side move from a retrusive, inferior and lateral position forward, upward, and inward [12,13,14]. In children, occlusion is approached directly from an opened mandibular position in the median sagittal plane. The movements of molar cusps on working and nonworking sides follow different paths in children and adults [15].

\subsection{Gender differences}

Women's and men's dietary habits differ considerably [16]. If the dynamics of the masticatory process are considered and correlated with gender, males perform faster and wider chewing cycles. The duration of a single chewing cycle is extended in females [17]. Obviously, males develop higher masticatory forces than women. What is noteworthy, however, is that it is not possible to infer any direct correlation between the masticatory force and muscle activity [18]. Nevertheless, many other factors such as age, dental status, prosthetic status overlap genderspecific differences in chewing [19]. In addition, health conditions, such as facial osteoporosis, may impair masticatory muscles patterns and performance [20]. 


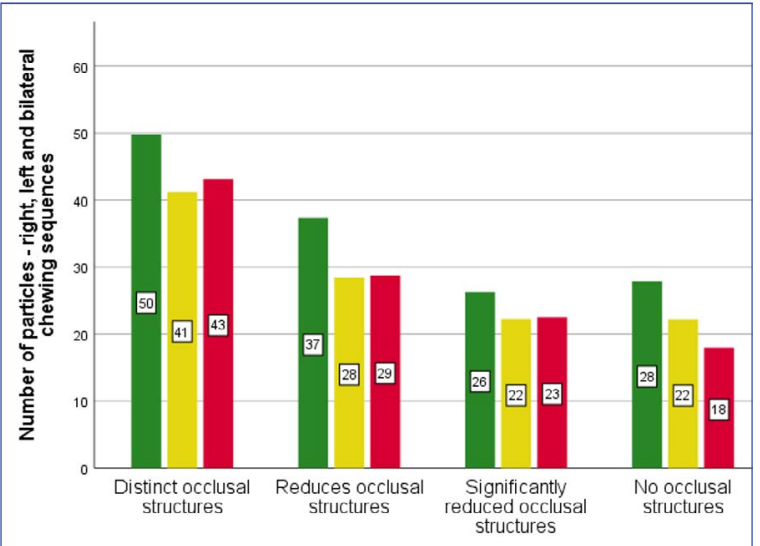

Figure 2a. Soft, medium and hard standard chewing test unit vs. overall occlusal quality.

\subsection{Chewing tests - requirements to be fulfilled}

A clinical test measuring chewing ability must consider extrinsic and intrinsic factors. Extrinsic parameters include food properties. Intrinsic parameters are among others age, gender, dental status. In addition, chewing tests units must be chosen that pose a similar challenge on the masticatory organ as natural foods.

1.4.1. Fragmentation methods as the golden standard

Significantly different methods to measure chewing ability are described in the specialized literature. Bearing in mind that mastication is intended to reduce food to as many equally sized particles as possible, methods that determine the degree of comminution are the methods of choice. SCTU is chewed within a defined time span and then spat out $[5,27]$. The number, area and distribution of the particles can be determined for analysis. An alternative method is to measure the time span required to achieve a certain degree of comminution. However, this requires several masticatory series of increasing duration to check when the degree of comminution is in line with the expectations $[12,27,28]$. The degree of comminution can be determined in various ways, among which the sieving method has become established as a valid model. Sieving methods are used to determine the distribution of the particle sizes of a granular material. This involves arranging several test sieves with decreasing mesh widths on top of one another. The quantity of the test material in each sieve can then be determined - for example by weighing the dried mass per sieve. In this way, conclusions can be drawn about the efficiency of the comminution process and hence the masticatory efficiency from the homogeneity of the particle size. This involves a consideration of the number of particles, the size of the particles and the ratio of the particle sizes in the food bolus. The methods for determining the masticatory ability based on the sieving technique principle are also referred to as fragmentation procedures [29]. Sieving methods are time consuming and not appropriate for routine

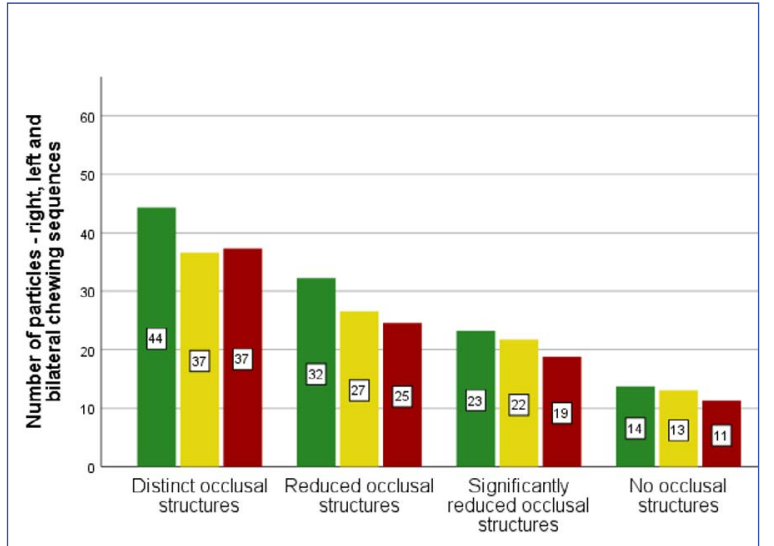

Figure 2b. Soft, medium and hard standard chewing test unit vs. overall occlusal quality in females.

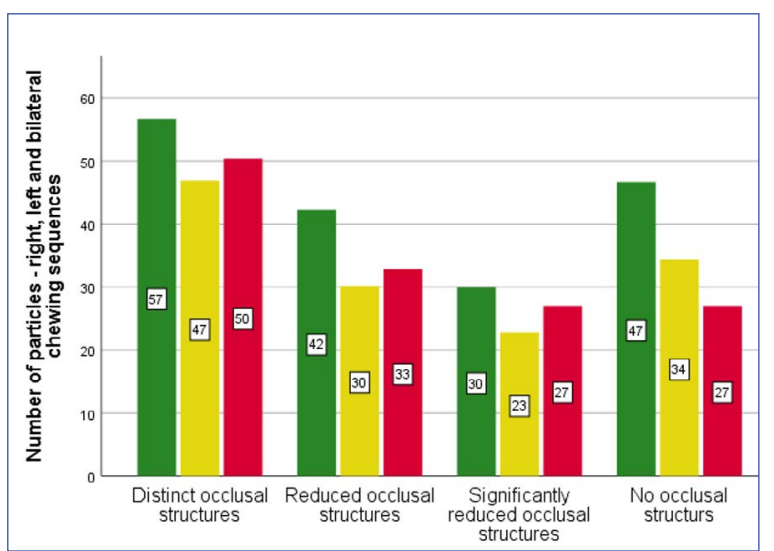

Figure 2c. Soft, medium and hard standard chewing test unit vs. overall occlusal quality in males.

clinical practice. Modern technologies such as optical scanning and/or imaging techniques can be used to overcome such burdens. The comparison of sieving with optical methods validates both methods and demonstrates comparable reliability and accuracy of information technology-based chewing fragmentation test procedures with sieving methods [30].

\subsection{Factors influencing chewing efficiency}

How well food is comminuted depends on many factors. Manifold parameters such as dental status, quality of chewing surface morphology, chewing forces, harmony of mandibular movements and co-ordination capacity of the neuromuscular system must be considered. These parameters are commonly subdivided into intrinsic (e.g. demographic attributes) and extrinsic (e.g. food properties) factors.

1.5.1. Intrinsic factors

Salivation and saliva quality affect the outcome of the masticatory sequence [31]. The degree of moisture of the food and in some cases additional fluid intake during mastication contribute to a change in the masticatory performance [32]. Age and gender are intrinsic modulators as well. All these factors affect mastication [33]. On the other hand, the effect of age may not be as pronounced as initially assumed. The number of masticatory cycles 


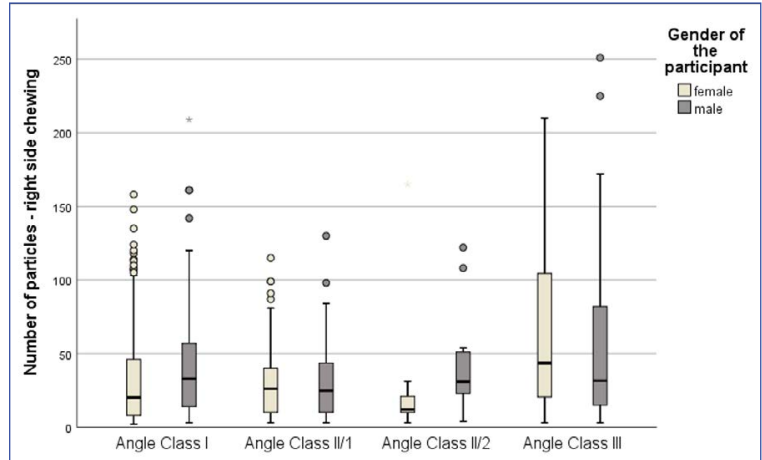

Figure 3a. Rigt side chewing efficiency vs. Angle classification on the right side; female vs. male.

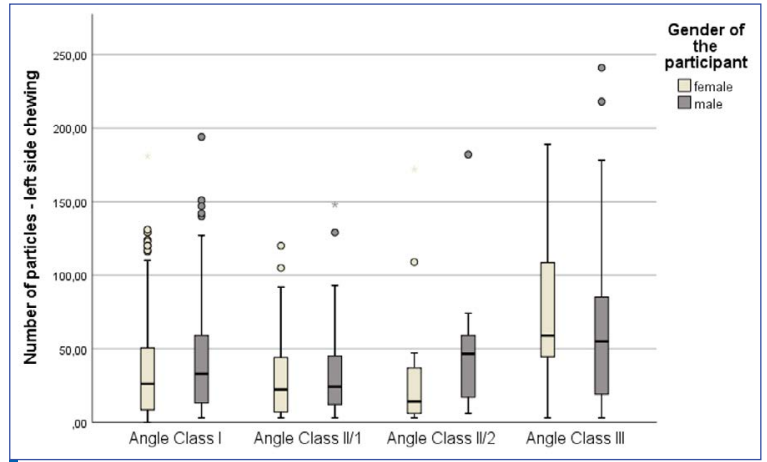

Figure 3b. Left side chewing efficiency vs. Angle classification on the left side; female vs. male.

Table 1. Overview of the perceived properties of food that have a significant influence on subjective perception and the so-called mouthfeel. The subjective taxation of food ingested in the mouth is by no means constant from the time it is placed in the mouth until it is swallowed, but can change considerably during a chewing sequence.

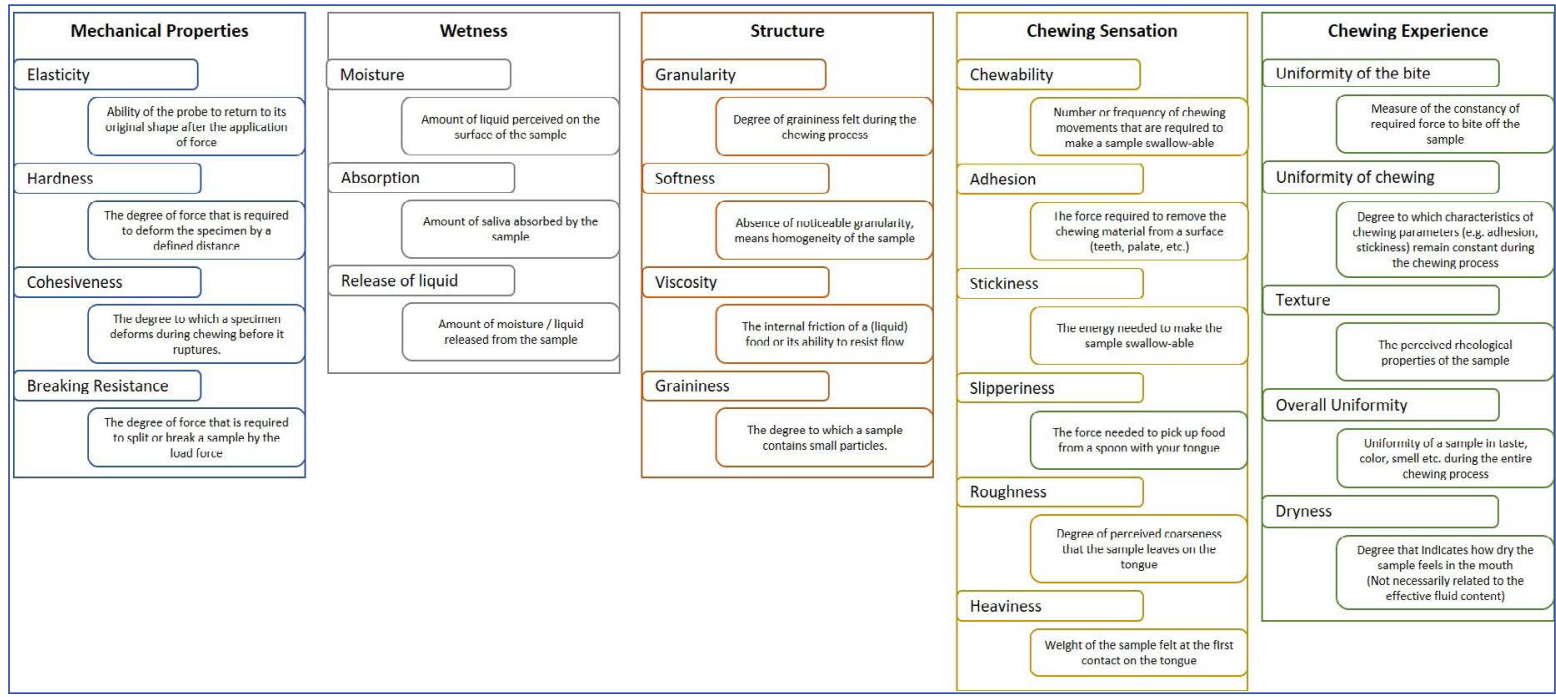

required to comminute a standardised food unit increases with age, but the capacity for individual adaptation to the properties of the food persists even in elderly subjects. The stomatognathic system remains relatively well preserved if people retain their dentition. This contrasts with other areas of the locomotor apparatus, which may be because the stomatognathic apparatus is used every day, even in people who already have difficulties walking [28]. 1.5.2. Extrinsic factors

Like the parameters attributable to the individual, food has a considerable effect on the process of mastication. The masticatory force, the vertical and lateral excursions of the masticatory movements, the rate of individual masticatory cycles and the frequency of mastication are parameters affected by food properties. The rheological properties of food are controlled by mechanisms localised in the brainstem $[2,21,22,24,34,35,36]$. Varying food textures can be used to influence dietary habits, such as more rapid or slower mastication, but in this case, hardness is not the most critical factor. Food properties such as plasticity, elasticity, and size have a direct effect on the afferent input signal in the central nervous system. Instead, parameters such as elasticity, springiness, texture, chewability and cohesiveness cause changes in masticatory behaviour. By changing food texture, it is possible to alter masticatory behaviour. The timespan, until a food bolus is swallowed, can be varied by food properties. Slower eating, which can be induced by a change in texture, thus produces a reduction in energy intake without affecting post-meal satisfaction [35].

Consensus exists to define the task of the masticatory process as the oral fragmentation of food to form a semi-liquid bolus for swallowing, a SCT should reflect on this: SCTU to be comminuted into as much and as equally sized particles as possible within a predefined length of the chewing sequence. Chewing media that do not degrade, such as chewing gum, do not meet this fundamental requirement [18]. Additionally, understanding chewing efficiency is not possible with one chewing sequence only (preferred chewing side problem). Intra-individual comparisons such as right vs. left vs. bilateral or soft vs. medium vs. hard based on several chewing sequences provides an in-depth review for clinicians and a better understanding for laypersons (patients and relatives). 


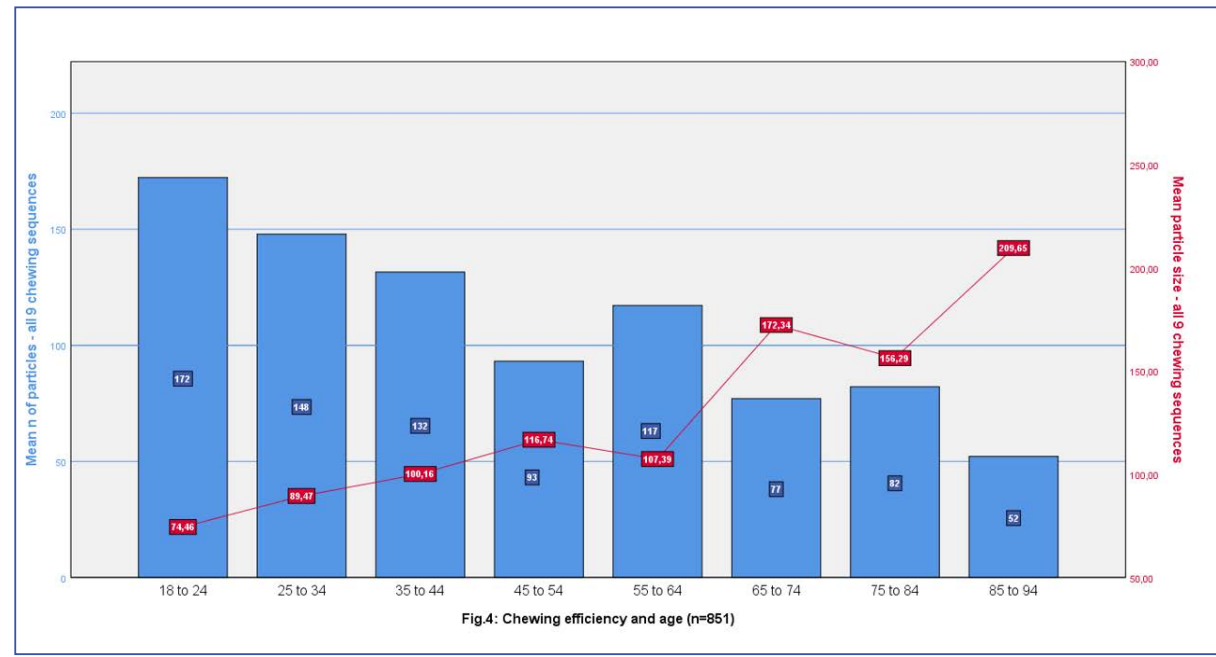

I Figure 4. Chewing efficiency and age $(n=851)$.

ITable 2. The categories to assess occlusal quality, using photo's for classification: no to distinct occlusal structures. The evaluation was performed per quadrant.

\begin{tabular}{|c|c|}
\hline Reference & Judgement \\
\hline \hline & no occlusal structures \\
\hline
\end{tabular}

\begin{tabular}{|l|l|}
\hline & $\begin{array}{c}\text { significantly reduces } \\
\text { occlusal structures }\end{array}$ \\
\hline
\end{tabular}

\begin{tabular}{|c|c|}
\hline & $\begin{array}{c}\text { reduced occlusal } \\
\text { structures }\end{array}$ \\
\hline & $\begin{array}{c}\text { distinct occlusal } \\
\text { structures }\end{array}$ \\
\hline
\end{tabular}

\subsection{Research question}

The role of the quality of occlusion as an influencing factor of masticatory efficiency is controversially discussed in the dental community. The aim of this article is to contribute to a better understanding of whether occlusion significantly influences chewing efficiency or not and to highlight possible cofounders such as angle classification, age, gender, treatment needs and intraoral status.

The null hypotheses tested are:

$\mathrm{H}_{0}$ 1: The distribution of number of particles (or mean area of particles, respectively) is the same across categories occlusal quality.

$\mathrm{H}_{0}$ 2: The distribution of number of particles (or mean area of particles, respectively) is the same across categories Angle classifications.

$\mathrm{H}_{0} 3$ : The distribution of number of particles (or mean area of particles, respectively) is the same across age groups.

$\mathrm{H}_{0}$ 4: The distribution of number of particles (or mean area of particles, respectively) is the same for females and males.

$\mathrm{H}_{0}$ 5: The subjective assessment of treatment needs by the investigator correlates with the distribution of number of particles (or mean area of particles, respectively).

$\mathrm{H}_{0} 6$ : The distribution of number of particles (or mean area of particles, respectively) is independent from the maxillary and mandibular status.

\section{METHODOLOGY}

The participants were instructed to try to break down the standardized chewing tests units (SCTU) into as many small pieces as possible. After 30 seconds the participants spited out the particles into a sieve. The particles were placed on a acquisition board with calibration elements. Standardised images are then taken and analysed using a validated software application. The surface areas of each of the particles are measured and displayed in $\mathrm{mm}^{2}$. The a priori determined Null Hypotheses were tested with the Kruskal-Wallis test followed by an applicable post hoc test with Bonferroni correction for repeated tests, to be rejected if $p \leq 0,01$. Data are collected as part of the BID-11400 study, multicentre and multinational clinical trial to evaluate masticatory functionality and quality of life. The study is approved by the Ethic Committee of University Cologne, Nr. 16-082, 15. May 2017. This evaluation includes data form projects which were recorded within the BID-11400 study, but solely by students as part of the Master of Science curriculum - Biomedical Interdisciplinary Dentistry at Steinbeis University Berlin. The data were collected between 2017-2019. A total of 851 data sets were used for these analyses.

Occlusal quality was judged by the investigator, using reference pictures, and determining occlusal quality for each quadrant (Tab. 2). The overall occlusal quality and the quality of occlusion on the right and left side were consolidated for the analytic process. Gender and year of birth were recorded for each participant. The age was calculated, and each 


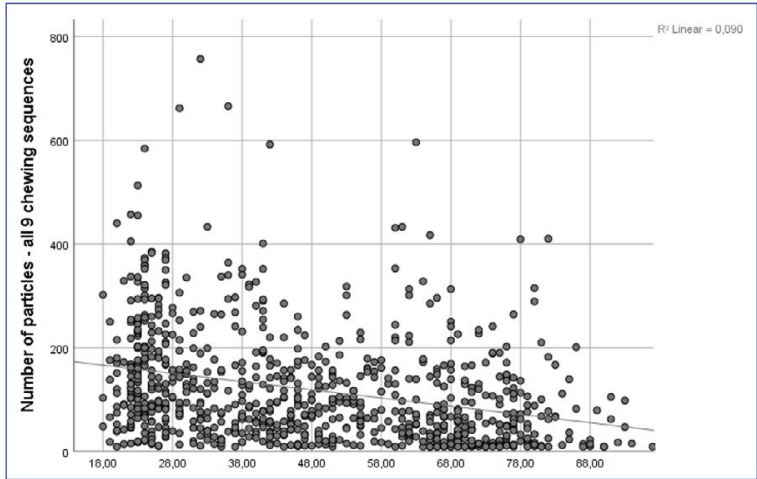

Figure 5a. Overall chewing efficiency vs. age; females vs. males.

participant was assigned to one age group: 18-24y; 25-34y; 35-44y; 45-54y; 55-64y; 65-74y; 75-84y. The treatment needs were assessed by the investigator. Theassessment was made in the following categories: clear indication for treatment; average treatment indication; minor treatment indication; no treatment indication. The maxillary and mandibular status was captured, for this analysis, only 3 categories have been used: Prevalence of natural teeth with minimal presence of restorations; Prevalence of natural teeth with the presence of fillings and partial crowns; Fixed prosthesis with bridges and crowns including implants. The chosen procedure should reflect the real clinical situation.

The standardized chewing test (SCT) was performed as follows: The patient is instructed on how to carry out the required nine masticatory sequences, each lasting 30 seconds. SCTU are used with tree different types of hardness. The basic mass for the SCTU is a standard formula used and approved commercially for fruit gum. The difference in hardness is achieved by the amount of gelatin added. Three different colors are used - algae extract for the green/soft, carrot/pumpkin extract for the yellow/medium and elder for the red/hard. The SCTU are equally flavored with strawberry essence. The chosen shape of the SFM is slightly cylindrical, with a diameter of $2 \mathrm{~cm}$ and a height of $1 \mathrm{~cm}[5,37]$.

The instruction to the patient is: "Try to break down the test item into as many small pieces as you can". The patient should chew in a concentrated way and should not be or get distracted while chewing. After 30 seconds the patient spits out the particles into a sieve. The particles per chewing sequence are placed on a recording plate with calibration elements and spread out so that the particles are at least $1 \mathrm{~mm}$ apart. Standardised images are then taken and analysed using a software application. The surface areas of each of the particles are measured and displayed in $\mathrm{mm}^{2}$. From this information the following key data can be determined: number $n$, area, and ratio of the particle sizes.

\section{RESULTS}

A sample of $n=842$ participants (female $n=460$, $54,6 \%$; male $n=382,45,4 \%$ ) performed a standardized

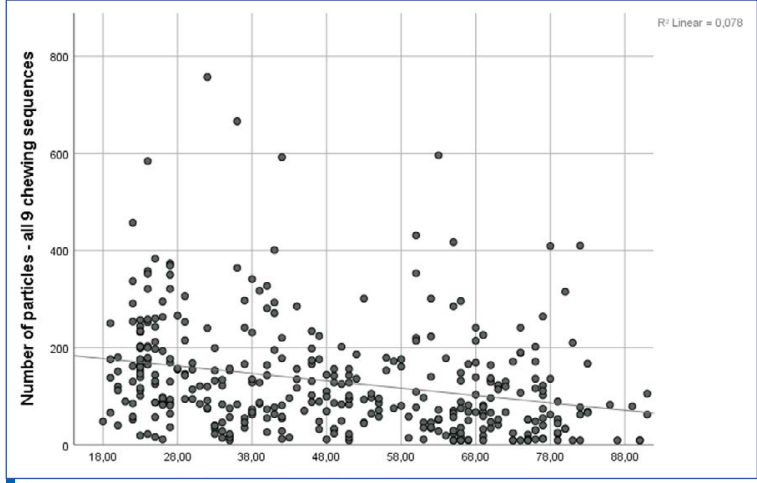

Figure 5b. Overall chewing efficiency vs. age; females.

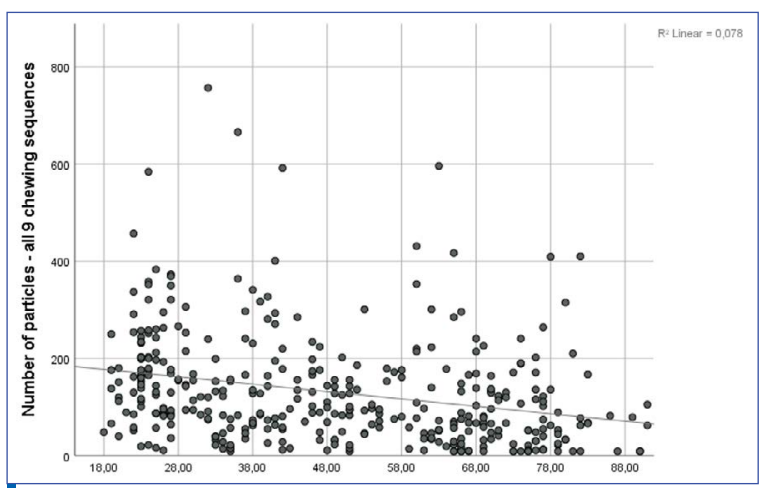

Figure 5c. Overall chewing efficiency vs. age; males.

chewing test (SCT). In addition, data on the participant's occlusal quality, angle classification, age, gender, treatment needs and intraoral status were recorded. The age distribution was as follows: $18-24 y(n=124,14,7 \%$; 26-34y $(n=160,19 \%) ; 35-44 y$ $(\mathrm{n}=126,15 \%) ; 45-54 \mathrm{y}(\mathrm{n}=110,13,1 \%) ; 55-64 \mathrm{y}(\mathrm{n}=84$, $10 \%) ; 65-74 y(n=131,15,6 \%) ; 75-84 y(n=85,10,1 \%)$, $>85 y(n=22,2,5 \%)$. Age group $>85 y$ was excluded for this analysis due to the low percentage.

\subsection{Quality of occlusion and Chewing efficiency}

The $\mathrm{H}_{0} 1$ "The distribution of number of particles (or mean area of particles, respectively) is the same across categories occlusal quality" was tested with a Kruskal-Wallis test followed by a post hoc test with Bonferroni correction for repeated tests. The difference between the occlusal categories was significant $(p<0,001)$. The post hoc analysis shows the marked difference between all four occlusal categories. This applies to overall chewing efficiency as well as for the right and left sides, where only the masticatory test results for that side were used for the analysis (Fig.1a,1b,1c). Considering occlusal quality, an almost linear reduction of the numbers of particles can be seen in especially in females, but not in males (Fig. 2a,2b,2c).

\subsection{Influence of Angle's classification on masticatory efficiency}

The $\mathrm{H}_{0} 2$ "The distribution of number of particles (or mean area of particles, respectively) is the same across categories Angle classifications" was tested using a Kruskal-Wallis test followed by a post hoc test 


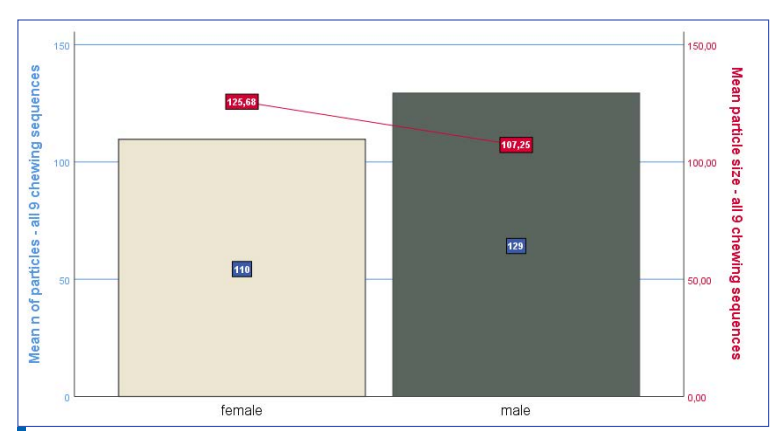

Figure 6. Overall chewing efficiency vs. gender.

with Bonferroni correction for repeated tests. The differences between the Angle classifications were significant $(p<0,001)$. The post hoc analysis showed that dental classes I, II/1 and II/2 do not differ. Angle's class III differs significantly from the other dental classes. This applies to both the right and left sides, where only the masticatory test results for that side were used for the analysis (Fig. 3a,3b). However, the results should be interpreted cautious since class II malocclusions were underrepresented in the study sample. Hence it cannot be confirmed that Angle's classification has a major impact on masticatory performance.

\subsection{Influence of Age on masticatory efficiency}

The $\mathrm{H}_{0} 3$ "The distribution of number of particles (or mean area of particles, respectively) is the same across age groups" was tested with a Kruskal-Wallis test $(p<0.001)$ followed by a post hoc test with Bonferroni correction for repeated tests. The results are presented in Table 3. The influence of age quoted in the literature can be confirmed based on the results of this study. It can be inferred that intraoral factors, especially in the elderly, significantly exceed the influencing factors age on chewing efficiency and should be taken into consideration in treatment strategies of an elderly population.

\subsection{Gender-specific differences}

The $\mathrm{H}_{0} 4$ "The distribution of number of particles (or mean area of particles, respectively) is the same for females and males" was tested with a MannWhitney-U test $(p<0.001)$. A SCT test based on nine chewing sequences shows that men generate markedly more (male 129 vs. female 110) and smaller particles (male $107.25 \mathrm{~mm}^{2}$ vs. female $125.68 \mathrm{~mm}^{2}$ ) than women (Fig.6). The difference is significant $(p=0.002$ for the number of particles, $p<0.001$ for the particle area). This overall difference between males and females can also be seen for soft and hard SCTU (Fig.7).

\subsection{Assessment of treatment needs and chewing efficiency}

The $\mathrm{H}_{0} 5$ "The subjective assessment of treatment needs by the investigator correlates with the distribution of number of particles (or mean area of

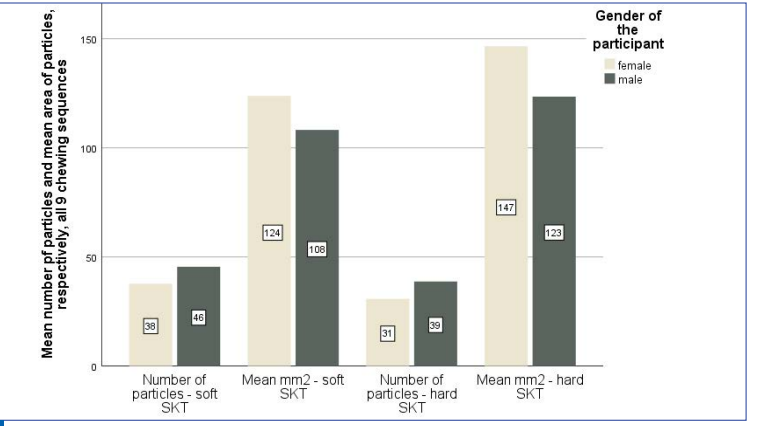

Figure 7. Influence of soft and hard standard chewing test units on chewing efficiency, per gender.

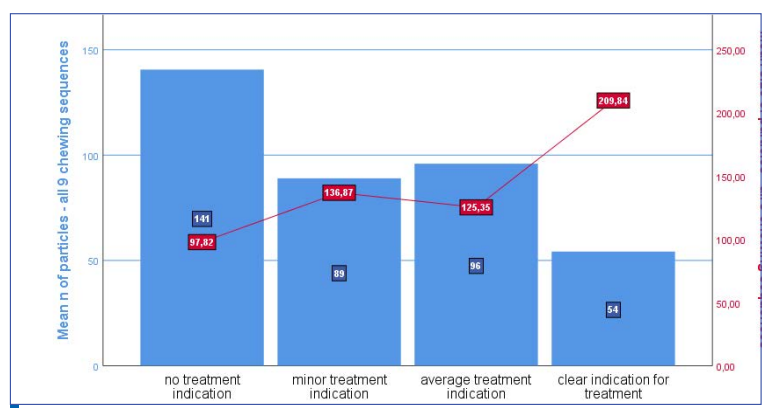

Figure 8. Chewing efficiency vs. assessment of treatment needs by investigators.

particles, respectively)" was tested with a KruskalWallis test $(p<0.001)$ followed by post hoc test with Bonferroni correction for repeated tests. The results are presented in Fig.8. The post hoc analysis showed that objective masticatory efficiency coincides with the assessment of treatment need for the extreme categories "no treatment indication" and "clear indication for treatment" only, while no significant difference $(p=0.515)$ was found between the "average" and "minor" treatment indication groups.

\subsection{Maxillary and mandibular status and masticatory efficiency}

The categories "Prevalence of natural teeth with minimal presence of restorations (upper jaw: $n=475$; lower jaw: $n=494)$ ), "Prevalence of natural teeth with the presence of fillings and partial crowns (upper jaw $\mathrm{n}=90$; lower jaw $\mathrm{n}=103$ )" and "Fixed prosthesis with bridges and crowns including implants (upper jaw $n=178$; lower jaw $n=155$ )" are incorporated in this analysis. The analysis was done separately for the upper and lower jaws. The $\mathrm{H}_{0} 6$ "The distribution of number of particles (or mean area of particles, respectively) is independent from the maxillary and mandibular status" was tested. The highest masticatory performance is achieved by natural teeth with minimal presence of restorations, followed by natural teeth with fillings and partial crowns. The results apply for upper (Fig.9a) and lower jaw (Fig.9b). Fixed prosthetic restorations such as crowns and bridges, including implant-supported fixed dentures, do not reach the results of natural occlusion. The Mann-Whitney- $U$ test was used to test for significance. For maxillary status no significant difference was found between "Prevalence of natural 


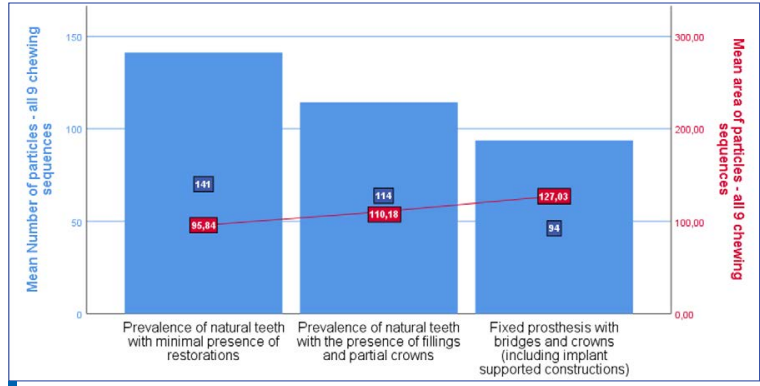

Figure 9a. Overall chewing efficiency vs. current status of the upper jaw (natural occlusal and fixed prosthesis only).

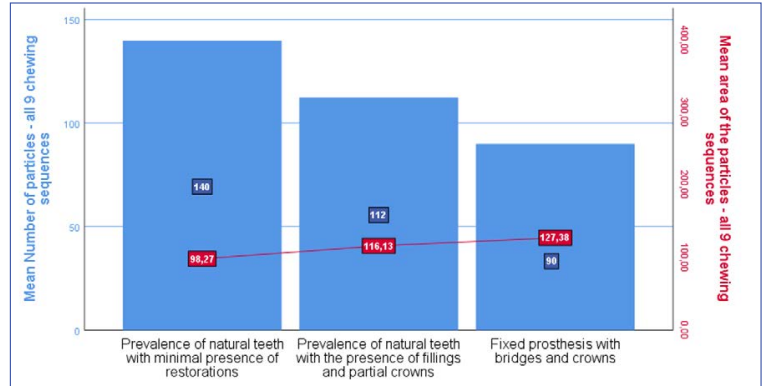

Figure 9b. Overall chewing efficiency vs. current status of the lower jaw (natural occlusal and fixed prosthesis only).

ITable 3. Results of a one-way ANOVA on ranks (Kruskal-Wallis test) followed by a post hoc test with Bonferroni correction for repeated tests to test whether chewing efficiency is different in age groups. In elderly, the age-related decline of chewing performance seems to be altered by other influencing intrinsic factors.

\begin{tabular}{|c|c|c|c|c|c|c|c|c|}
\hline $18-24$ years & $18-24$ years & & & & & & & \\
\hline $25-34$ years & 1,000 & $25-34$ years & & & & & & \\
\hline $35-44$ years & 0,023 & 1,000 & $35-44$ years & & & & & \\
\hline $45-54$ years & $<0,001$ & 0,005 & 1,000 & $45-54$ years & & & & \\
\hline $55-64$ years & 0,004 & 0,425 & 1,000 & 1,000 & $55-64$ years & & & \\
\hline $65-74$ years & $<0,001$ & $<0,001$ & $<0,001$ & 0,040 & 1,000 & $65-74$ years & & \\
\hline $75-84$ years & $<0,001$ & $<0,001$ & 0,012 & 1,000 & 0,413 & 1,000 & $75-84$ years & \\
\hline $85-94$ years & $<0,001$ & $<0,001$ & 0,013 & 0,094 & 0,384 & 1,000 & 1,000 & $85-94$ years \\
\hline
\end{tabular}

teeth with minimal presence of restorations" and "Prevalence of natural teeth with the presence of fillings and partial crowns". A significant difference was found between "Prevalence of natural teeth with minimal presence of restorations" and "Fixed prosthesis with bridges and crowns including implants" $(p<0.001)$. A significant difference was found between "Prevalence of natural teeth with the presence of fillings and partial crowns" and "Fixed prosthesis with bridges and crowns including implants" $(p<0.039)$.

\section{DISCUSSION}

The chewing surface morphology is admirably adapted to the food of each species $[1,4,8]$. Individuals with compromised occlusion have an impaired chewing efficiency compared to those with satisfactory occlusion $[2,10,37]$. Categorizing occlusion into four groups-distinct occlusal structures; reduced occlusal structures; significantly reduces occlusal structures; no occlusal structures - and correlating these groups with the masticatory performance reveals the significant impact of occlusal morphology on masticatory efficiency (Fig.1a). These results support the influence of occlusion on the function of mastication and the chewing efficiency. A well-formed and distinctly shaped occlusal morphology significantly increases masticatory efficiency.

The angle's classification is usually applied to describe occlusion. And, although critical and noncoherent, the Angle classification is often used to deduce functional conditions. In addition, the terms norm-occlusion and malocclusion are often used equated to functional or dysfunctional occlusion.
Maxillary and mandibular teeth are best aligned with each other in a class I occlusion, assuming regular tooth shapes. What is known as a class II or class III malocclusion does not exist in primates - this would obviously be a distinct disadvantage in the search for food and consumption of food. Malocclusion seems to conflict with the evolutionary concept of survival of the fittest. In humans, malocclusion has ceased to be a selection criterion because hunting and cutting tools are available and fire allows to preprocess meals.

The masticatory performance diminishes with age $[19,28]$. In particular, the length of the masticatory sequence required to produce the same particle size increases. Peyron et al. [28] quantified this increase of the required time for chewing with three masticatory cycles per ten years of life on average. Consequently, a $50 \%$ increase in numbers of chewing cycles needed to reach the same size of the particles between 25years and 75years [28]. These data can, to a certain extent, be transferred to the result of a chewing test based on fragmentation procedures. As the masticatory sequences are always the same length of 30 seconds, the number of chewed particles decreases with age.

Coincidentally, the mean particle size of chewed particles increases with age. The results show a continuous decrease in number of particles produced by elderly (Fig. 4). The decrease in 10 years can be calculated with approximately $15 \%$. Undoubtedly, the decrease in masticatory performance is no longer linear from the age of about 50 years. A possible explanation for this finding is a progressively intraoral alteration - including an increasing number of missing teeth, more and extended dentures and reduced structures of occlusal surfaces. The decline 
in the masticatory performance with age follows a similar path for both genders (Fig. 5). Gender-specific differences are described in the literature [16,18,32]. Forces during chewing are higher in males than females. The masticatory movements of men are also executed with greater vertical and transverse amplitude $[6,12,13,14]$. The overall results confirm the difference in masticatory efficiency between men and women that is quoted in the literature. But, as the difference is similar for soft and hard SCTU, it cannot be inferred that males are much more effective in chewing the hard SCTU than females. This result only partly confirms the fundamental assumption that, because of higher physical force, males are better able to masticate than women.

The clinical occlusal appearance is often used as treatment indicator. The subjective assessment of treatment needs and an objective measure of masticatory performance should coincide. This required the researchers to make a subjective assessment of treatment need without using further tests or other data. The assessment was made in the following categories: clear indication for treatment; average treatment indication; minor treatment indication; no treatment indication. The post hoc analysis showed that the objective masticatory efficiency coincides with the assessment of treatment need for the extreme categories "no treatment indication" and "clear indication for treatment" only, while no significant difference $(p=0.515)$ was found between the "average" and "minor" treatment indication groups. Therefore, the purely subjective assessment of treatment need cannot be considered to be adequate in individual decision making.

The aim of oral rehabilitation is to maintain and restore all functions of the masticatory organ. A clear relationship between intraoral status and masticatory efficiency can be deduced by the scientific literature $[2,10,11,19,25,27,32]$. It is still unclear what prosthetic rehabilitation best restores the individual's masticatory function.

The masticatory performance is often debriefed only via questionnaires and self-reporting by patient. The question arises if prosthetic rehabilitation can reach the chewing efficiency of natural occlusion? In this particular analysis, only natural occlusion and fixed prosthodontics are included. Based on the analysed tests of the SCT, it can be concluded, that fixed prosthodontic rehabilitation are not capable to reach the functional efficiency of natural occlusion.

\section{CONCLUSION}

The better the masticatory performance, the more particles will be found in the lower size ranges. Conversely, it can be concluded that few and large particles denote insufficient masticatory performance. The golden standard for testing chewing performance is the sieve technique. But, the clinical efforts in performing a chewing test based on the sieving method exceed the resources in daily practice. Therefore, contemporary IT-technologies including optical methods may be implemented in clinical routine, as such techniques have been demonstrated to be reliable and accurate to analyse chewing fragmentation test procedures comparably with sieving methods. Within the limitations of this study, it can be concluded:

- The quality of occlusion has a significant impact of chewing efficiency. The masticatory performance depends on the natural and/or artificial chewing surface morphology.

- Angle classification explains chewing efficiency only to some extent. A reasoning of an individual functional status just from Angle's classification is critical.

- Age is an important intrinsic factor which influences masticatory performance. But, at least in the age groups above 60 years, the intraoral status seems to overrule the influence of age. The maintenance of a sufficient functional oral status is a crucial task in care of the aging population.

- Gender differences in chewing outcomes, as described in the literature, can be confirmed by the results of this study. It can be added that the quality of occlusion affects both genders in a similar way. Higher muscle forces in males are not able to compensate poor occlusal quality.

- The assessment of treatment needs by the clinician based on a clinical inspection correlates with the masticatory performance in clear conditions only - either no treatment needs or clear indication for treatment. If the judgement of the treatment needs is unclear - minor or average treatment indications - the chewing efficiency is not correlated.

- Fixed prosthodontics, including implant supported construction, do not reach the chewing performance of natural occlusion with minor restorations.

\section{CONFLICT OF INTEREST}

There is a conflict of interest: The author is Founder and CEO of OREHAB-Minds GmbH, DE-70567 Stuttgart, Germany.

\section{AUTHOR CONTRIBUTIONS}

GS contributed to the concept, protocol, data gathering and analysis, their interpretation and critically revising the manuscript.

\section{ACKNOWLEDGMENTS}

None. 


\section{REFERENCES}

1. Lucas PW, Luke DA. Chewing it over: basic principles of food breakdown. In: Chivers DJ, Wood BA, Bilsborough A. (eds) Food acquisition and processing in primates. Boston, MA: Springer Since; 1984. https://doi.org/10.1007/978-1-4757-5244-1_12

Google Scholar

2. Slavicek G. Human mastication. J Stomat Occ Med. 2010;3:29-

41. https://doi.org/10.1007/s12548-010-0044-6.

3. Geissler CA, Bates JF. The nutritional effects of tooth loss. Am J

Clin Nutr. 1984;39(3):478-489. doi:10.1093/ajcn/39.3.478.

Google Scholar

4. Ungar PS. Mammalian dental function and wear: a review. Biosurf Biotribol. 2015;1(1):25-41. https://doi.org/10.1016/j.

bsbt.2014.12.001.

[Crossref] Google Scholar

5. Slavicek G, Soykher M, Soykher M, et al. Relevance of a standard food model in combination with electronic jaw movement recording on human mastication pattern analysis. $A d v$ Biosci Biotechnol. 2010;1(2):68-78. doi:10.4236/abb.2010.12011.

[Crossref] Google Scholar

6. Gibbs $\mathrm{CH}$, Mahan $\mathrm{PE}$, Lundeen $\mathrm{HC}$, et al. Occlusal forces during chewing-influences of biting strength and food consistency.

J Prosthet Dent 1981:46(5):561-567. doi:10.1016/0022

3913(81)90247-x

[Full text links] [Crossref] [PubMed] Google Scholar Scopus WoS

7. Butler PM. Evolution and mammalian dental morphology. J Biol

Buccale. 1983;11(4):285-302.

[PubMed] Google Scholar Scopus WoS

8. Orthlieb JD. The curve of Spee: understanding the sagittal

organization of mandibular teeth. Cranio. 1997;15(4):333-340. doi

$: 10.1080 / 08869634.1997 .11746028$

[Full text links] [Crossref] [PubMed] Google Scholar Scopus WoS

9. Bortun CM, Rusu LC. Recording mastication angels by Planas's laws. Stoma Edu J. 2014;1 (2):86-91. https://doi.org/10.25241/ stomaeduj.2014.1(2).art.1

[Crossref]

10. Meyer GB, Bernhardt O, Constantinescu MV. Fundamentals of occlusion and masticatory function. Stoma Edu J. 2014;1(2):116122. https://doi.org/10.25241/stomaeduj.2014.1(2).art.6.

[Crossref]

11. Tandetzki J. [Development of a practical test to measure chewing efficiency on patients]. Masterthese Department for Interdisziplinary Dentristry and Technologie University of Continuing Education Krems, 2011.

12. Gibbs CH, Wickwire NA, Jacobson AP, et al. Comparison of typical chewing patterns in normal children and adults. J Am Dent Assoc. 1982;105(1):33-42. doi:10.14219/jada. archive.1982.0073.

[Full text links] [Crossref] [PubMed] Google Scholar Scopus WoS 13. Julien KC, Buschang PH, Throckmorton GS, Dechow

PC. Normal masticatory performance in young adults and children. Arch Oral Biol. 1996;41(1):69-75. doi:10.1016/00039969(95)00098-4

[Full text links] [Crossref] [PubMed] Google Scholar Scopus WoS 14. Gibbs CH, Lundeen HC, Mahan PE, Fujimoto J. Chewing movements in relation to border movements at the first molar. J Prosthet Dent. 1981;46(3):308-322. doi:10.1016/00223913(81)90220-1.

[Full text links] [Crossref] [PubMed] Google Scholar Scopus

15. Gibbs $\mathrm{CH}$, Mahan PE, Lundeen $\mathrm{HC}$, et al. Occlusal forces during chewing--influences of biting strength and food consistency. J Prosthet Dent. 1981;46(5):561-567. doi:10.1016/0022-3913(81)90247-x.

[Full text links] [Crossref] [PubMed] Google Scholar Scopus WoS 16. Shiga H, Kobayashi Y, Katsuyama H, et al. Gender difference in masticatory performance in dentate adults. J Prosthodont Res. 2012;56(3):166-169. doi:10.1016/j.jpor.2012.02.001.

[Full text links] [Crossref] [PubMed] Google Scholar Scopus WoS 17. Uesugi H, Shiga H. Relationship between masticatory performance using a gummy jelly and masticatory movement. J Prosthodont Res. 2017:61(4):419-425. doi:10.1016/j. jpor.2017.01.001.

[Full text links] [Crossref] [PubMed] Google Scholar Scopus WoS 18. Youssef RE, Throckmorton GS, Ellis E 3rd, Sinn DP. Comparison of habitual masticatory patterns in men and women using a custom computer program. J Prosthet Dent. 1997;78(2):179-186. doi:10.1016/s0022-3913(97)70123-9.

[Full text links] [Crossref] [PubMed] Google Scholar Scopus WoS 19. Mishellany-Dutour A, Renaud J, Peyron MA, et al. Is the goal of mastication reached in young dentates, aged dentates and aged denture wearers?. Br J Nutr. 2008;99(1):121-128. doi:10.1017/ S0007114507795284.

[Full text links] [Crossref] [PubMed] Google Scholar

20. Siéssere $S$, Sousa LG, Lima Nde A, et al. Electromyographic activity of masticatory muscles in women with osteoporosis. Braz Dent J. 2009·20(3):237-342 doi:10.1590/s0103-

64402009000300012

[Full text links] [Crossref] [PubMed] Google Scholar Scopus 21. Woda A, Foster K, Mishellany A, Peyron MA. Adaptation of healthy mastication to factors pertaining to the individual or to the food. Physiol Behav. 2006;89(1):28-35. doi:10.1016/j. physbeh.2006.02.013.

[Full text links] [Crossref] [PubMed] Google Scholar Scopus 22. Kohyama K, Sasaki T, Hayakawa F. Characterization of food physical properties by the mastication parameters measured by electromyography of the jaw-closing muscles and mandibular kinematics in young adults. Biosci Biotechnol Biochem. 2008;72(7):1690-1695. doi:10.1271/bbb.70769.

[Full text links] [Crossref] [PubMed] Google Scholar Scopus WoS

23. van der Bilt A, Olthoff LW, van der Glas HW, et al. A

mathematical description of the comminution of food

during mastication in man. Arch Oral Biol. 1987;32(8):579-586.

doi:10.1016/0003-9969(87)90067-7.

[Crossref] [PubMed] Google Scholar Scopus WoS

24. Peyron MA, Mishellany A, Woda A. Particle size distribution of food boluses after mastication of six natural foods. J Dent Res. 2004:83(7):578-582. doi:10.1177/154405910408300713.

[Full text links] [Crossref] [PubMed] Google Scholar Scopus WoS 25. Slagter AP, Olthoff LW, Steen WH, Bosman F. Comminution of food by complete-denture wearers. J Dent Res. 1992;71(2):380386. doi:10.1177/00220345920710020601.

[Full text links] [PubMed] Google Scholar

26. Woda A, Nicolas E, Mishellany-Dutour A, et al. The masticatory normative indicator. J Dent Res. 2010;89(3):281-285. doi:10.1177/0022034509357022.

Google Scholar

27. Helkimo E, Carlsson GE, Helkimo M. Chewing efficiency and state of dentition. A methodologic study. Acta Odontol Scand. 1978;36(1):33-41. doi:10.3109/00016357809026364.

[Full text links] [Crossref] [PubMed] Google Scholar Scopus 28. Peyron MA, Blanc O, Lund JP, Woda A. Influence of age on adaptability of human mastication. J Neurophysiol. 2004:92(2):773-779. doi:10.1152/jn.01122.2003.

[Full text links] [Crossref] [PubMed] Google Scholar Scopus WoS 29. Schneider G, Senger B. Clinical relevance of a simple fragmentation model to evaluate human masticatory performance. J Oral Rehabil. 2002:29(8):731-736. doi:10.1046/ j.1365-2842.2002.00967.x

[Full text links] [Crossref] [PubMed] Google Scholar Scopus WoS 30. Mowlana F, Heath MR, Van der Bilt A, Van der Glas HW. Assessment of chewing efficiency: a comparison of particle size distribution determined using optical scanning and sieving of almonds. J Oral Rehabil. 1994;21(5):545-551. doi:10.1111/j.1365-2842.1994.tb01168.x.

[Full text links] [Crossref] [PubMed] Google Scholar Scopus WoS 31. van der Bilt A, Engelen L, Pereira LJ, et al. Oral physiology and mastication. Physiol Behav. 2006;89(1):22-27. doi: 10.1016/j. physbeh.2006.01.025

Google Scholar

32. Pereira LJ, Gavião MB, Engelen L, Van der Bilt A. Mastication and swallowing: influence of fluid addition to foods. J Appl Oral Sci. 2007;15(1):55-60. doi:10.1590/s1678-77572007000100012. [Full text links] [Crossref] [PubMed] Google Scholar Scopus WoS 33. van der Bilt A. Assessment of mastication with implications for oral rehabilitation: a review. J Oral Rehabil. 2011;38(10):754780. doi:10.1111/j.1365-2842.2010.02197.x.

[Full text links] [Crossref] [PubMed] Google Scholar Scopus WoS 34. Foster KD, Woda A, Peyron MA. Effect of texture of plastic and elastic model foods on the parameters of mastication. $J$ Neurophysiol. 2006;95(6):3469-3479. doi:10.1152/jn.01003.2005. [Full text links] [Crossref] [PubMed] Google Scholar Scopus WoS 35. Wee MSM, Goh AT, Stieger M, Forde CG. Correlation of instrumental texture properties from textural profile analysis (TPA) with eating behaviours and macronutrient composition for a wide range of solid foods. Food Funct. 2018;9(10):5301-5312. doi:10.1039/c8fo00791h.

[Full text links] [Crossref] [PubMed] Google Scholar WoS 36. Witt T, Stokes JR. Physics of food structure breakdown and bolus formation during oral processing of hard and soft solids. Curr Opin Food Sci. 2015;(3):110-117. https://doi.org/10.1016/j. cofs.2015.06.011.

Google Scholar

37. Slavicek G, Soykher M, Gruber H. et al. A novel standard food model to analyze the individual parameters of human mastication. J Stomat Occ Med. 2009;2:163-174. https://doi. org/10.1007/s12548-009-0029-5.

[Crossref] Google Scholar 


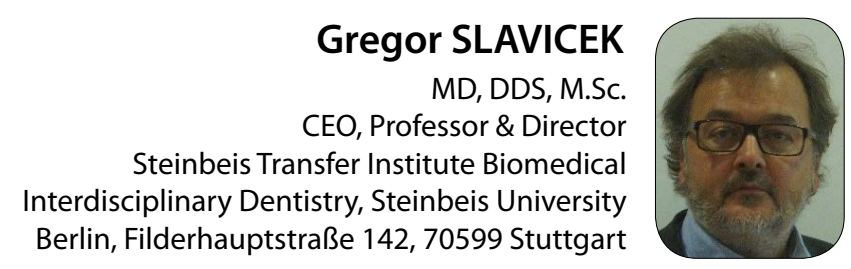

Dr. Slavicek is an MD, specialized in Dentistry. He is currently Director of the Steinbeis Transfer Institute Biomedical Interdisciplinary Dentistry, Steinbeis University Berlin. Since 2019, he has been CEO of Orehab Minds GmbH in Stuttgart, Germany. He graduated from the University Vienna (medicine and dentistry), also specializing in Clinical Research at the same university (Master of Science). He attended additional postgraduate orthodontic training at University Aarhus (Denmark), Prof. B. Melsen, and postgraduate gnathological training at University of Florida (USA), Prof. H. Lundeen and Prof. C. Gibbs. He is an honorary member of the Italian Gnathological Society. He received an honorary professorship from the Ukrainian Dental Society. He is an honorary member of the Italian Gnathological Society. He was visiting professor at the first medical state University in Moscow Sechenov (2014-2018).

\title{
Questions
}

\section{Which of the following statement is incorrect?}

$\square$ a. Chewing efficiency is related to angle classification only to a certain extent;

ab. Chewing efficiency increases with age;

ac. Chewing efficiency with males and females is significantly different;

$\square d$. Chewing efficiency is dependent on the prosthetic rehabilitation.

\section{Which of the following parameters can influence chewing efficiency?}

\author{
$\square$ a. Chewing surface morphology; \\ ab. Gender; \\ ac. Age; \\ d. Answers 1-3 are correct.
}

\section{A subjective assessment of treatment needs by the clinician?}

$\square$ a. Can be considered as a sufficient method to understand individual masticatory functions;

b. Is able to distinguish even small differences in treatment needs;

ac. Is not possible to distinguish even in extreme situations of treatments needs (not treatment indication vs. clear treatment indication);

$\square$ d. A subjective assessment should be proven by suitable tests.

\section{An adequate chewing function test for clinical use?}

$\square$ a. Is based on the principles of the sieving method;

b. Considers the ability of the stomatognathic system to fragment food;

ac. Contemporary IT-supported methods based on the evaluation of standardized photos are qualitatively equivalent to the sieving method;

$\square$ d. Answers 1-3 are correct. 